\title{
ON SAMPLING EXPANSIONS OF KRAMER TYPE
}

\author{
ANTHIPPI POULKOU
}

Received 31 October 2002

We treat some recent results concerning sampling expansions of Kramer type. The link of the sampling theorem of Whittaker-Shannon-Kotelnikov with the Kramer sampling theorem is considered and the connection of these theorems with boundary value problems is specified. Essentially, this paper surveys certain results in the field of sampling theories and linear, ordinary, first-, and second-order boundary value problems that generate Kramer analytic kernels. The investigation of the first-order problems is tackled in a joint work with Everitt. For the second-order problems, we refer to the work of Everitt and Nasri-Roudsari in their survey paper in 1999. All these problems are represented by unbounded selfadjoint differential operators on Hilbert function spaces, with a discrete spectrum which allows the introduction of the associated Kramer analytic kernel. However, for the first-order problems, the analysis of this paper is restricted to the specification of conditions under which the associated operators have a discrete spectrum.

\section{Introduction}

This paper surveys certain results in the area of sampling theories and linear, ordinary, first- and second-order boundary value problems that produce Kramer analytic kernels.

1.1. Notations. The symbol $\mathbf{H}(U)$ represents the class of Cauchy analytic functions that are holomorphic (analytic and regular) on the open set $U \subseteq \mathbb{C}$, that is, $\mathbf{H}(\mathbb{C})$ represents the class of all entire or integral functions on $\mathbb{C}$. The symbol $I=(a, b)$ denotes an arbitrary open interval of $\mathbb{R}$; the use of "loc" restricts a property to compact subintervals of $\mathbb{R}$. All the functions $f:(a, b) \rightarrow \mathbb{C}$ are taken to be Lebesgue measurable on $(a, b)$, all integrals are in the sense of Lebesgue, and AC denotes absolute continuity with respect to Lebesgue measure.

If $w$ is a weight function on $I$, then the Hilbert function space $L^{2}(I ; w)$ is the set of all complex-valued, Lebesgue measurable functions $f: I \rightarrow \mathbb{C}$ such that $\int_{a}^{b} w|f|^{2} \equiv$ $\int_{a}^{b} w(x)|f(x)|^{2} d x<+\infty$ and then, with due regard to equivalence classes, the norm and 
inner product are given by

$$
\|f\|_{w}^{2}:=\int_{I} w|f|^{2}, \quad(f, g)_{w}:=\int_{a}^{b} w(x) f(x) \bar{g}(x) d x .
$$

1.2. The W.S.K. sampling theorem. This sampling theorem owes its first appearance to Whittaker, in 1915. The same result was obtained later and independently by Kotel'nikov, in 1933, and by Shannon, in 1949. So, it is presently known in the mathematical literature as the W.S.K. theorem (see $[31,35,40]$ ). However, there are more names who have legitimate claims to be included and for a historical review, we refer to [26, 27]. Turning to the seminal paper by Shannon, this theorem, the proof of which is found in [35], reads as follows.

THEOREM 1.1 (see [35]). If a signal (function) $f(t)$ contains no frequencies higher than $W / 2$ cycles per second, that is, is band limited to $[-\pi W, \pi W]$, which means that $f(t)$ is of the form

$$
f(t)=\int_{-\pi W}^{\pi W} g(x) \exp (i x t) d x
$$

then $f(t)$ is completely determined by giving its ordinates at a sequence of points spaced $1 / W$ apart and $f(t)$ is the sum of its "scaled" cardinal series

$$
f(t)=\sum_{n=-\infty}^{\infty} f\left(\frac{n}{W}\right) \frac{\sin \pi(W t-n)}{\pi(W t-n)} .
$$

Remark 1.2. This is the first of the sampling theory results; the signal $f$ cannot change to a substantially new value in a time less than half a cycle of its highest frequency, $W / 2$ cycles per second. And moreover, the collection of "samples" $\{f(n / W): n=0, \pm 1, \pm 2, \ldots\}$ specifies $g$ via its Fourier series, since the general Fourier coefficient of $g$ (in (1.2)) is $f(n / W)$, and then $g$ specifies $f$ via (1.2). So, if $f$ can be "measured" at the sampling points $\{n / W: n \in \mathbb{Z}\}$, which are equidistantly spaced over the whole real line $\mathbb{R}$, then $f$ can be reconstructed uniquely at every point of the real line $\mathbb{R}$. The engineering principle established in this way leads to the assertion that certain functions whose frequency content is bounded are equivalent to an information source with discrete time. This has a major application in signal analysis, and in order to obtain, in general, a great appreciation of the broad scope of sampling theory, we refer, for example, to $[4,5,6,26,28,30,33]$.

The contents of the paper are as follows: Section 2 gives an analytical background information about the original and the analytic form of the Kramer theorem followed by a discussion concerning quasidifferential problems and operators; Section 3 gives an account of results with respect to the generation of Kramer analytic kernels from first-order boundary value problems, but without mentioning the spectral properties that yield a discrete spectrum of the associated operators; and finally, Section 4 deals with results about the connection of second-order linear ordinary boundary value problems and the Kramer sampling theorem. 


\section{Introduction to the analytical background}

2.1. The original and the analytic form of the Kramer theorem. In 1959, Kramer published the following remarkable result, the proof of which is given in [32].

Theorem 2.1 (Kramer theorem). Suppose that $f(t):=\int_{I} K(x, t) g(x) d x, t \in \mathbb{R}$, for some $g \in L^{2}(I)$, where $I$ is an open interval of $\mathbb{R}$ and the kernel $K: I \times \mathbb{R} \rightarrow \mathbb{R}$ satisfies the properties that, for each real $t, K(\cdot, t) \in L^{2}(I)$, and there exists a countable set of reals $\left\{t_{n}: n \in \mathbb{Z}\right\}$ such that $\left\{K\left(\cdot, t_{n}\right): n \in \mathbb{Z}\right\}$ forms a complete orthogonal set on $L^{2}(I)$. Then

$$
f(t)=\sum_{n \in Z} f\left(t_{n}\right) S_{n}(t), \quad S_{n}(t):=\frac{\int_{I} K(x, t) \bar{K}\left(x, t_{n}\right) d x}{\int_{I}\left|K\left(\cdot, t_{n}\right)\right|^{2} d x} .
$$

And moreover, the conditions on the kernel are met by certain solutions of selfadjoint eigenvalue problems, where the parameter $t$ is an eigenvalue parameter; the eigenvalues are chosen to be the sampling points and the complete orthogonal system of eigenfunctions, the set of functions $\left\{K\left(x, \lambda_{n}\right): n \in \mathbb{Z}\right\}$.

Remark 2.2. (i) Each eigenvalue problem that produces a complete set of eigenfunctions and also real simple and countably infinite many eigenvalues is suitable for the Kramer theorem. For a study of Kramer kernels constructed from boundary value problems, see, for example, $[7,32]$.

(ii) A certain class of boundary value problems transforms the W.S.K. sampling theorem (Theorem 1.1) into a particular case of the Kramer theorem. For example, take under consideration the selfadjoint, regular eigenvalue problem, for $\sigma>0, \lambda \in \mathbb{R}$ :

$$
-i y^{\prime}(x)=\lambda y(x), \quad x \in[-\sigma, \sigma], y(-\sigma)=y(\sigma) .
$$

The eigenvalues are given by $\lambda_{n}=n \pi / \sigma, n \in \mathbb{Z}$, and the corresponding eigenfunctions are $y_{n}(x)=\exp (i n \pi x / \sigma), n \in \mathbb{Z}$. The general solution $K(x, \lambda)=\exp (i x \lambda)$ of the differential equation is a suitable kernel for Theorem 1.1. So, if $f$ is of the form

$$
f(\lambda)=\int_{-\sigma}^{\sigma} \exp (i x \lambda) g(x) d x, \quad g \in L^{2}(-\sigma, \sigma), \lambda \in \mathbb{R},
$$

then there exists the sampling representation

$$
f(\lambda)=\sum_{n \in \mathbb{Z}} f\left(\frac{n \pi}{\sigma}\right) \frac{\sin (\sigma \lambda-n \pi)}{(\sigma \lambda-n \pi)} .
$$

(iii) The Kramer kernel that arises from the above example has a significant property. This property also emerges in a number of other cases of symmetric boundary value problems and is not predicted in the statement of Kramer's theorem, that is, $K(x, \cdot) \in$ $\mathbf{H}(\mathbb{C}), x \in I$ (see Section 1.1). For additional details of the previous boundary value problem, see the results in [15, Section 5.1].

The following theorem gives an analytic form of the Kramer theorem in the way that allows analytic dependence of the kernel on the sampling parameter. 
Theorem 2.3. Let $I=(a, b)$ be an arbitrary open interval of $\mathbb{R}$ and let $w$ be a weight function on $I$. Let the mapping $K: I \times \mathbb{C} \rightarrow \mathbb{C}$ satisfy the following properties:

(1) $K(\cdot, \lambda) \in L^{2}(I ; w)(\lambda \in \mathbb{C})$

(2) $K(x, \cdot) \in \mathbf{H}(\mathbb{C})(x \in(a, b))$,

(3) there exists a sequence $\left\{\lambda_{n} \in \mathbb{R}: n \in \mathbb{Z}\right\}$ satisfying

(i) $\lambda_{n}<\lambda_{n+1}(n \in \mathbb{Z})$,

(ii) $\lim _{n \rightarrow \pm \infty} \lambda_{n}= \pm \infty$,

(iii) the sequence of functions $\left\{K\left(\cdot, \lambda_{n}\right): n \in \mathbb{Z}\right\}$ forms a locally linearly independent and a complete orthogonal set in the Hilbert space $L^{2}(I ; w)$,

(4) the mapping $\lambda \mapsto \int_{a}^{b} w(x)|K(x, \lambda)|^{2} d x$ is locally bounded on $\mathbb{C}$.

Define the set of functions $\{K\}$ as the collection of all functions $F: L^{2}(I ; w) \times \mathbb{C} \rightarrow \mathbb{C}$ determined by, for $f \in L^{2}(I ; w)$,

$$
F(f ; \lambda) \equiv F(\lambda):=\int_{a}^{b} w(x) K(x, \lambda) f(x) d x \quad(\lambda \in \mathbb{C})
$$

Then for all $F \in\{K\}$,

(a) $F(f, \cdot) \in \mathbf{H}(\mathbb{C})\left(f \in L^{2}(I ; w)\right)$;

(b) if $S_{n}: \mathbb{C} \rightarrow \mathbb{C}$ is defined by, for all $n \in \mathbb{Z}$,

$$
S_{n}(\lambda):=\left\|K\left(\cdot, \lambda_{n}\right)\right\|_{w}^{-2} \int_{a}^{b} w(x) K(x, \lambda) \bar{K}\left(x, \lambda_{n}\right) d x \quad(\lambda \in \mathbb{C})
$$

then $S_{n} \in \mathbf{H}(\mathbb{C})$;

(c) $F(f, \lambda) \equiv F(\lambda)=\sum_{n \in \mathbb{Z}} F\left(\lambda_{n}\right) S_{n}(\lambda)$, for all $F \in\{K\}$, where the series is absolutely convergent, for each $\lambda \in \mathbb{C}$, and locally uniformly convergent on $\mathbb{C}$.

Proof. For the proof of this theorem see [18, Theorem 2 and Corollary 1]; the ideas for these results come from [10] and [21, Theorem 1.1].

Remark 2.4. (i) Suitable problems for the above theorem are, for example, regular selfadjoint eigenvalue problems of $n$ th-order and singular selfadjoint problems of second-order in the limit-circle endpoint case (for classifications of eigenvalue problems, see [34], and for information concerning Kramer analytic kernels, see, e.g., [15, 19, 41]).

(ii) As outlined in Remark 2.2(ii), the W.S.K. theorem can be seen as a particular case of Kramer's result for a certain class of problems. So, the question arises whether these two theorems are equivalent to each other or not. The link of the W.S.K. "sampling results" and Kramer's theorem has been the concern of many authors. The first person who dealt with this problem was Campbell in 1964 (see [7]). Later, there is a lot to be found in the literature; see, for example, $[29,42]$. Also, an extensive historical perspective of the equivalence of Kramer and W.S.K. theorems for second-order boundary value problems is given in [24]; there also may be found some results for the Bessel and the general Jacobi cases. 
2.2. Quasidifferential problems and operators. The environment of the general theory of quasiderivatives is the best for the study of symmetric (selfadjoint) boundary value problems which, as noticed in Remark 2.4(i), are a source for the generation of Kramer analytic kernels. Furthermore, all the classical differential expressions appear as special cases of quasidifferential expressions; for confirmation, we refer to [13, 14, 20, 25, 34]. Finally, the Shin-Zettl quasidifferential expressions are considered to be the most general ordinary linear differential expressions so far defined, for order $n \in \mathbb{N}$ and $n \geq 2$; for details see $[9,11,22,23,36,37,38,43]$. Accordingly, the general formulation of quasidifferential boundary value problems will be performed as follows.

Let $I=(a, b)$ be an open interval of the real line $\mathbb{R}$. Let $M_{n}$ be a linear ordinary differential expression. In the classical case, $M_{n}$ is of finite order $n \geq 1$ on $I$ with complex-valued coefficients, and of the form

$$
M_{n}[f]=p_{n} f^{(n)}+p_{n-1} f^{(n-1)}+\cdots+p_{1} f^{\prime}+p_{0} f
$$

where $p_{j}: I \rightarrow \mathbb{C}$ with $p_{j} \in L_{\mathrm{loc}}^{1}(I), j=0,1, \ldots, n-1, n$, and further $p_{n} \in \mathrm{AC}_{\mathrm{loc}}(I)$ with $p_{n}(x) \neq 0$, for almost all $x \in I$. For the special case $n=1$, see details in [12].

In the more general quasidifferential case, the expression $M_{n}$ is defined as in [23] and [14, Section I]. For $n \geq 2$, the expression $M_{n}:=M_{A}$ is determined by a complex Shin-Zettl matrix $A=\left[a_{r s}\right] \in Z_{n}(I)$ with the domain $D\left(M_{n}\right)$ of $M_{A}$ defined by

$$
\begin{gathered}
D\left(M_{A}\right):=\left\{f: I \longrightarrow \mathbb{C}: f_{A}^{[r-1]} \in \operatorname{AC}_{\mathrm{loc}}(I), \text { for } r=1,2, \ldots, n\right\}, \\
M_{A}[f]:=i^{n} f_{A}^{[n]}\left(f \in D\left(M_{A}\right)\right),
\end{gathered}
$$

where the quasiderivatives $f_{A}^{[j]}$, for $j=1,2, \ldots, n$, are taken relative to the matrix $A \in$ $Z_{n}(I)$. For these results and additional properties, see the notes [9]. In this investigation, $M_{A}$ is Lagrange symmetric in the notation of $[9,20]$.

Every classical ordinary linear differential expression $M_{n}$, as in (2.7), can be written as a quasidifferential expression $M_{A}$, as in (2.8), with the same order $n \geq 2$. The first-order differential expressions are essentially classical in form. Therefore, we can assume that when $n \geq 2, M_{n}$ is a quasidifferential expression specified by an appropriate Shin-Zettl matrix $A \in Z_{n}(I)$. When $n=1$, we consider $M_{1}$ as a classical expression and the analysis given here works also in this case.

Now, the Green's formula for $M_{n}$ has the form

$$
\int_{\alpha}^{\beta}\left\{\bar{g} M_{n}[f]-f \overline{M_{n}[g]}\right\}=[f, g](\beta)-[f, g](\alpha) \quad\left(f, g \in D\left(M_{n}\right)\right),
$$

for any compact subinterval $[\alpha, \beta]$ of $(a, b)$. Here the skew-symmetric sesquilinear form $[\cdot, \cdot]$ is taken from (2.9); that is, it maps $D\left(M_{n}\right) \times D\left(M_{n}\right) \rightarrow \mathbb{C}$ and is defined, for $n \geq 2$, by

$$
[f, g](x):=i^{n} \sum_{r=1}^{n}(-1)^{r-1} f^{[n-r]}(x) \overline{g^{(r-1)}}(x) \quad\left(x \in(a, b), f, g \in D\left(M_{n}\right)\right)
$$


and, for $n=1$, by

$$
[f, g](x):=i \rho(x) f(x) \bar{g}(x) \quad\left(x \in(a, b), f, g \in D\left(M_{1}\right)\right) .
$$

From the Green's formula (2.9), it follows the limits

$$
\begin{aligned}
& {[f, g](a):=\lim _{x \rightarrow a^{+}}[f, g](x),} \\
& {[f, g](b):=\lim _{x \rightarrow b^{-}}[f, g](x),}
\end{aligned}
$$

both exist and are finite in $\mathbb{C}$.

The spectral differential equations associated with the pairs $\left\{M_{n}, w\right\}$, where $w$ is a given nonnegative weight (see Section 1.1), are

$$
M_{n}[y]=\lambda w y \quad \text { on }(a, b)
$$

with the spectral parameter $\lambda \in \mathbb{C}$. The solutions of (2.13) are considered in the Hilbert function space $L^{2}((a, b) ; w)$ (see Section 1.1). In order to define symmetric boundary value problems in this space, linear boundary conditions of the form (see (2.9), (2.10), (2.11), and (2.12))

$$
\left[y, \beta_{r}\right] \equiv\left[y, \beta_{r}\right](b)-\left[y, \beta_{r}\right](a)=0, \quad r=1,2, \ldots, d,
$$

have to be connected, where the family $\left\{\beta_{r}, r=1,2, \ldots, d\right\}$ is a linearly independent set of maximal domain functions chosen to satisfy the symmetry condition

$$
\left[\beta_{r}, \beta_{s}\right](b)-\left[\beta_{r}, \beta_{s}\right](a)=0 \quad(r, s=1,2, \ldots, d) .
$$

The integer $d \in \mathbb{N}_{0}$ is the common deficiency index of (2.13) determined in $L^{2}((a, b) ; w)$ and gives the number of boundary conditions needed for the boundary value problem $((2.13),(2.14))$ to be symmetric, that is, to produce a selfadjoint operator in $L^{2}((a, b) ; w)$. This boundary value problem generates a uniquely determined unbounded selfadjoint operator $T$ in the space $L^{2}((a, b) ; w)$; see [23].

If the problem is regular on an interval $(a, b)$, in which case this interval has to be bounded, then $d=n$ and the generalized boundary conditions (2.14) require the pointwise values of the solution $y$ and its quasiderivatives at the endpoints $a$ and $b$. For this regular case when the order $n=2 m$ is even and the Lagrange symmetric matrix is real valued, see details in [34]. In the case $n=1$, the index $d$ can take the values 0 or 1 , but the value 0 is rejected (see Remark 3.3). In the case $n=2$, essentially the Sturm-Liouville case, the index $d$ may take the values 0,1 , or 2 ; this value depends on the regular/limitpoint/limit-circle classification, in $L^{2}(I ; w)$, at the endpoints $a$ and $b$ of the differential expression $M_{n}$ (cf. [39, Chapter II]).

For the connection between the classical and quasidifferential systems, we refer to [14].

\section{First-order problems}

In this section, we investigate in greater details the link between the Kramer sampling theorem and linear ordinary differential equations of first-order. The results we present 
here are given in [19]. We only point out that the development of our operator theory as a source for the construction of Kramer analytic kernels is not given here; see [19] for details of these Kramer kernels. The operator theory required is to be found in [1, 2, 8]; for the classical theory of selfadjoint extensions of symmetric operators as based on Hilbert space constructions, see [34].

3.1. Differential equations and operators. The selfadjoint boundary value problems considered here are generated by the general first-order Lagrange symmetric linear differential equation which defines the differential expression $M_{1}$ and is of the form

$$
\begin{aligned}
M_{1}[y](x): & =i \rho(x) y^{\prime}(x)+\frac{1}{2} i \rho^{\prime}(x) y(x)+q(x) y(x) \\
& =\lambda w(x) y(x), \quad \forall x \in(a, b),
\end{aligned}
$$

where $-\infty \leq a<b \leq+\infty$ and $\lambda \in \mathbb{C}$ is the spectral parameter. Also,

$$
\begin{gathered}
\rho, q, w:(a, b) \longrightarrow \mathbb{R}, \\
\rho \in \mathrm{AC}_{\mathrm{loc}}(a, b), \quad \rho(x)>0, \quad \forall x \in(a, b), \\
q, w \in L_{\text {loc }}^{1}(a, b), \\
w(x)>0, \quad \text { for almost all } x \in(a, b) .
\end{gathered}
$$

Under conditions (3.2), the differential equation (3.1) has the following initial value properties; let $c \in(a, b)$ and $\gamma \in \mathbb{C}$, then there exists a unique mapping $y:(a, b) \times \mathbb{C} \rightarrow \mathbb{C}$ with

(i) $y(\cdot, \lambda) \in \operatorname{ACloc}_{\text {loc }}(a, b)$, for all $\lambda \in \mathbb{C}$,

(ii) $y(x, \cdot) \in \mathbf{H}$, for all $x \in(a, b)$,

(iii) $y(c, \lambda)=\gamma$, for all $\lambda \in \mathbb{C}$,

(iv) $y(\cdot, \lambda)$ satisfies $(3.1)$, for almost all $x \in(a, b)$ and all $\lambda \in \mathbb{C}$.

However, direct formal integration shows that the required solution $y$ is given by

$$
y(x, \lambda)=\gamma \sqrt{\frac{\rho(c)}{\rho(x)}} \exp \left(\int_{c}^{x} \frac{(\lambda w(t)-q(t))}{i \rho(t)} d t\right), \quad \forall x \in(a, b), \forall \lambda \in \mathbb{C} .
$$

Remark 3.1 (see [19, Lemma 2.1]). A necessary and sufficient condition to ensure that the solution $y(\cdot, \lambda) \in L^{2}((a, b) ; w)$, for all $\lambda \in \mathbb{C}$, is

$$
\int_{a}^{b} \frac{w(t)}{\rho(t)} d t<+\infty
$$

We notice that if there are any selfadjoint operators $T$ in $L^{2}((a, b) ; w)$ generated by $M_{1}$ (see (3.1)), then all such operators have to satisfy the inclusion relation

$$
T_{0} \subseteq T=T^{*} \subseteq T_{1}=T_{0}^{*}
$$


where $T_{0}$ and $T_{1}$ are the minimal and maximal operators, respectively, generated by $M_{1}$. From the general theory of unbounded operators in Hilbert space, such selfadjoint operators exist if and only if the deficiency indices $\left(d^{-}, d^{+}\right)$of $T_{0}$ are equal; see [34, Chapter IV]. Thus for selfadjoint extensions of $T_{0}$ to exist, there are only two possibilities:

(i) $d^{-}=d^{+}=0$,

(ii) $d^{-}=d^{+}=1$.

Remark 3.2 (see [19, Lemma 4.1]). (i) The indices $d^{-}=d^{+}=0$ if and only if, for some $c \in(a, b), w / \rho \notin L^{1}(a, c]$ and $w / \rho \notin L^{1}[c, b)$.

(ii) The indices $d^{-}=d^{+}=1$ if and only if $w / \rho \in L^{1}(a, b)$.

Remark 3.3. (a) In the case of Remark 3.2(i), if we define the operator $T$ by $T:=T_{0}^{*}=T_{0}$, then $T$ is the (unique) selfadjoint operator in $L^{2}((a, b) ; w)$ generated by the differential expression $M_{1}$ of (3.1). The selfadjoint boundary value problem, in this case, consists only of the differential equation (3.1). In fact, the spectrum of $T$ is purely continuous and occupies the whole real line, that is, $\sigma(T)=C \sigma(T)=\mathbb{R}$. We note that this case can give no examples of interest for sampling theories. As an example in $L^{2}(-\infty,+\infty)$, consider $i y^{\prime}(x)=\lambda y(x)$, for all $x \in(-\infty,+\infty)$.

(b) In the case of Remark 3.2(ii), which covers all regular cases of (3.1) and all singular cases when condition (3.4) is satisfied, the general Stone/von Neumann theory of selfadjoint extensions of closed symmetric operators in Hilbert space proves that there is a continuum of selfadjoint extensions $\{T\}$ of the minimal operator $T_{0}$ with $T_{0} \subset T \subset T_{1}$. These extensions can be determined by the use of the generalized Glazman-Krein-Naimark (GKN) theory for differential operators as given in [12, Section 4, Theorem 1]. The domain of any selfadjoint extension $T$ of $T_{0}$ can be obtained as a restriction of the domain of the maximal operator $T_{1}$. These restrictions are obtained by choosing an element $\beta \in D\left(T_{1}\right)$ such that $\beta$ arises from a nonnull member of the quotient space $D\left(T_{1}\right) / D\left(T_{0}\right)$ with the symmetric property $($ recall $(2.15))[\beta, \beta]\left(b^{-}\right)-[\beta, \beta]\left(a^{+}\right)=0$. With this boundary condition function $\beta \in D\left(T_{1}\right)$, the domain $D(T)$ is now determined by

$$
D(T):=\left\{f \in D\left(T_{1}\right):[f, \beta]\left(b^{-}\right)-[f, \beta]\left(a^{+}\right)=0\right\},
$$

and the selfadjoint operator is defined by $T f:=w^{-1} M[f]$, for all $f \in D(T)$.

For an example of such a boundary condition function $\beta$, see $[19$, Section $4,(4.20)]$.

Now, the selfadjoint boundary value problem consists of considering the possibility of finding nontrivial solutions $y(\cdot, \lambda)$ of the differential equation $(3.1)$ with the property $y(\cdot, \lambda) \in L^{2}((a, b) ; w)$ that satisfies the boundary condition

$$
[y(\cdot, \lambda), \beta]\left(b^{-}\right)-[y(\cdot, \lambda), \beta]\left(a^{+}\right)=0
$$

The solution of this problem depends upon the nature of the spectrum $\sigma(T)$ of the selfadjoint operator $T$ determined by the choice of the boundary condition element $\beta$.

In the case of Remark 3.2(ii), it is shown in [19, Theorem 5.1] that the spectrum of $\sigma(T)$ of any selfadjoint extension $T$ of $T_{0}$ is discrete, simple, and has equally spaced eigenvalues on the real line of the complex spectral plane. 
3.2. Kramer analytic kernels. The results in [19] read as follows.

Theorem 3.4. Suppose that (3.1) satisfies (3.2) and also (3.4) to give equal deficiency indices $d^{-}=d^{+}=1$. Let the selfadjoint operator $T$ be determined by imposing a coupled boundary condition (3.6) on the domain $D\left(T_{1}\right)$ of the maximal operator $T_{1}$ using a symmetric boundary condition function $\beta$ as in Remark 3.3(b). Denote the spectrum $\sigma(T)$ of $T$ by $\left\{\lambda_{n}: n \in \mathbb{Z}\right\}$. Define the mapping $K:(a, b) \times \mathbb{C} \rightarrow \mathbb{C} b y$, where $c \in(a, b)$ is fixed,

$$
K(x, \lambda):=\frac{1}{\sqrt{\rho(x)}} \exp \left(\int_{c}^{x} \frac{\lambda w(t)-q(t)}{i \rho(t)} d t\right), \quad \forall x \in(a, b), \lambda \in \mathbb{C} .
$$

Then the kernel $K$, together with the point set $\left\{\lambda_{n}: n \in \mathbb{Z}\right\}$, satisfies all the conditions required for the application of Theorem 2.3 to yield $K$ as a Kramer analytic kernel in the Hilbert space $L^{2}((a, b) ; w)$.

Proof. See [19].

For an example of this general result, we refer to [19, Theorem 7.1] (cf. Remark 2.2(ii)). This example is considered in [15] too.

\section{Second-order problems}

In this section, we deal with the generation of Kramer analytic kernels from second-order linear ordinary boundary value problems. The results given here can be found in [15].

4.1. Sturm-Liouville theory. Sturm-Liouville boundary value problems are effective in generating Kramer analytic kernels. These problems concern the classic Sturm-Liouville differential equation

$$
-\left(p(x) y^{\prime}(x)\right)^{\prime}+q(x) y(x)=\lambda w(x) y(x) \quad(x \in I=(a, b)),
$$

where $-\infty \leq a \leq b \leq+\infty$ and $\lambda \in \mathbb{C}$ is the spectral parameter. Also,

$$
\begin{gathered}
p, q, w:(a, b) \longrightarrow \mathbb{R}, \\
p^{-1}, q, w \in L_{\text {loc }}^{1}(a, b), \\
w(x)>0, \quad \text { for almost all } x \in(a, b) .
\end{gathered}
$$

For a discussion on the significance of these conditions, see [16, page 324]. For the general theory of Sturm-Liouville boundary value problems, see [39, Chapters I and II]. Accordingly, we impose a structural condition.

Condition 4.1. The endpoint $a$ of the differential equation (4.1) is to be regular or limitcircle in $L^{2}(I ; w)$; independently, the endpoint $b$ is to be regular or limit-circle in $L^{2}(I ; w)$ (cf. [21]).

Remark 4.2. The endpoint classification of Condition 4.1 leads to a minimal, closed, symmetric operator in $L^{2}(I ; w)$ generated by $(4.1)$ with deficiency indices $d^{ \pm}=2$; in turn, this 
requires that all selfadjoint extensions $A$ of this minimal, symmetric operator are determined by applying two linearly independent, symmetric boundary conditions and either

(i) both conditions are separated with one applied at $a$ and with one applied at $b$, or

(ii) both conditions are coupled.

4.1.1. Regular or limit-circle case with separated boundary conditions. This case of Condition 4.1 and Remark 4.2(i) concerns the results of [21]. The Sturm-Liouville differential equation is given by (4.1) and satisfies (4.2). The separated boundary conditions are

$$
\left[y, \kappa_{-}\right](a)=\left[y, \kappa_{+}\right](b)=0
$$

where, for a given pair of functions $\left\{\mathcal{\kappa}_{-}, \chi_{-}\right\}$, the following conditions are fulfilled:

(C1) $\kappa_{-}, \chi_{-}:(a, b) \Rightarrow \mathbb{R}$ are maximal domain functions,

(C2) $\left[\kappa_{-}, \chi_{-}\right](a)=1$.

The pair $\left\{\kappa_{+}, \chi_{+}\right\}$satisfies analogous conditions at the endpoint $b$.

This symmetric boundary value problem gives a selfadjoint differential operator $T$ with the following properties:

(a) $T$ is unbounded in $L^{2}((a, b) ; w)$,

(b) the spectrum of $T$ is real and discrete with limit points at $+\infty$ or $-\infty$ or both,

(c) the spectrum of $T$ is simple,

(d) the eigenvalues and eigenvectors satisfy the boundary value problem.

The results in [21] are given by the following theorem.

Theorem 4.3. Let the coefficients $p, q$, and $w$ satisfy the conditions (4.2); let the SturmLiouville quasidifferential equation (4.1) satisfy the endpoint classification of Condition 4.1; let the separated boundary conditions be given by (4.3), where the boundary condition functions $\left\{\kappa_{-}, \chi_{-}\right\}$and $\left\{\kappa_{+}, \chi_{+}\right\}$satisfy conditions (C1) and (C2); let the selfadjoint differential operator $T$ be determined by the separated, symmetric boundary value problem; let the simple, discrete spectrum of $T$ be given by $\left\{\lambda_{n}: n \in \mathbb{Z}\right\}$ with $\lim _{n \rightarrow \pm \infty} \lambda_{n}= \pm \infty$; let $\left\{\psi_{n}: n \in \mathbb{Z}\right\}$ be the eigenvectors of $T$; and let the pair of basis solutions $\left\{\phi_{1}, \phi_{2}\right\}$ of (4.1) satisfy the initial conditions, for some point $c \in(a, b)$ :

$$
\begin{array}{ll}
\phi_{1}(c, \lambda)=1, & \left(p \phi_{1}^{\prime}\right)(c, \lambda)=0, \\
\phi_{2}(c, \lambda)=0, & \left(p \phi_{2}^{\prime}\right)(c, \lambda)=1 .
\end{array}
$$

Define the analytic Kramer kernel $K_{-}:(a, b) \times \mathbb{C} \rightarrow \mathbb{C}$ by

$$
K_{-}(x, \lambda):=\left[\phi_{1}(\cdot, \lambda), \kappa_{-}\right](a) \phi_{2}(x, \lambda)-\left[\phi_{2}(\cdot, \lambda), \kappa_{-}\right](a) \phi_{1}(x, \lambda) .
$$

Then

(i) $K_{-}(\cdot, \lambda)$ is a solution of $(4.1)$, for all $\lambda \in \mathbb{C}$, and $K_{-}(\cdot, \lambda) \in \mathbb{R}(\lambda \in \mathbb{R})$;

(ii) $K_{-}(\cdot, \lambda)$ is an element of the maximal domain and in particular of $L^{2}((a, b) ; w)$;

(iii) $\left[K_{-}(\cdot, \lambda), \kappa_{-}\right](a)=0$;

(iv) $\left[K_{-}(\cdot, \lambda), \kappa_{+}\right](b)=0$ if and only if $\lambda \in\left\{\lambda_{n}: n \in \mathbb{Z}\right\}$;

(v) $K_{-}(x, \cdot) \in \mathbf{H}(\mathbb{C})(x \in(a, b))$; 
(vi) $K_{-}\left(\cdot, \lambda_{n}\right)=k_{n} \psi_{n}$, where $k_{n} \in \mathbb{R} \backslash\{0\}(n \in \mathbb{Z})$;

(vii) $K_{-}$is unique up to multiplication by a factor $e(\cdot) \in \mathbf{H}(\mathbb{C})$ with $e(\lambda) \neq 0(\lambda \in \mathbb{C})$ and $e(\lambda) \in \mathbb{R}(\lambda \in \mathbb{R})$.

Remark 4.4. The notation $K_{-}$is chosen for technical reasons; there is a kernel $K_{+}$with similar properties, but with $a$ and $\kappa_{-}$replaced by $b$ and $\kappa_{+}$.

For an example, we refer to [15, Section 5.2, Example 5.8]. This example can also be found in [21].

4.1.2. Regular or limit-circle case with coupled boundary conditions. This case of Condition 4.1 and Remark 4.2(ii) covers the results of [16]. Here the situation is different. Let (4.1) satisfy (4.2) and let the boundary conditions be given by

$$
\mathbf{y}(b)=e^{i \alpha} T \mathbf{y}(a), \quad \text { for some } \alpha \in[-\pi, \pi]
$$

with the $2 \times 2$ matrix $T=\left[t_{r s}\right]$, where $t_{r s} \in \mathbb{R}(r, s=1,2)$, $\operatorname{det}(T)=1$, and the $2 \times 1$ vector $\mathrm{y}$ is defined by

$$
\mathbf{y}(t):=\left(\begin{array}{l}
{[y, \theta](t)} \\
{[y, \phi](t)}
\end{array}\right) \quad(t \in(a, b))
$$

$T$ is called boundary condition matrix. The functions $\theta$ and $\phi$ are chosen such that

(i) $\theta$ and $\phi$ are real-valued maximal domain functions;

(ii) $[\theta, \phi](a)=\lim _{t \rightarrow a^{+}}[\theta, \phi](t)=1$;

(iii) $[\theta, \phi](b)=\lim _{t \rightarrow b^{-}}[\theta, \phi](t)=1$.

For example, $\theta$ and $\phi$ can be real-valued solutions of $(4.1)$ on $(a, b)$.

The boundary conditions (4.6) are coupled and selfadjoint, for each endpoint either regular or limit-circle, and are in canonical form (see [3]).

Let the pair of basis solutions $\{u, v\}$ of the differential equation (4.1) be specified by the possibly singular initial conditions (cf. [3]), for all $\lambda \in \mathbb{C}$,

$$
\begin{array}{lll}
{[u, \theta](a, \lambda)=0,} & {[u, \phi](a, \lambda)=1,} \\
{[v, \theta](a, \lambda)=1,} & {[v, \phi](a, \lambda)=0 .}
\end{array}
$$

To define a differential operator $A$, choose any boundary condition matrix $T$ and any $\alpha \in[-\pi, \pi]$; the boundary value problem gives a selfadjoint differential operator with the properties (a), (b), and (d) and, in place of (c), the property that the multiplicity of the spectrum is either 1 or 2 .

Remark 4.5. For complex boundary conditions, that is, when $0<\propto<\pi$ or $-\pi<\propto<0$, the spectrum is always simple. In the case of real boundary conditions, that is, $\alpha=-\pi, 0, \pi$, the spectrum may or may not be simple (see [3]).

The complex case. According to the comments made in Remark 4.5, the results in [16] are divided into two parts. The first part is referred to as the complex case when $0<\alpha<\pi$ or $-\pi<\alpha<0$ and this gives the following theorem. 
Theorem 4.6. Let (4.1) satisfy Condition 4.1, where the coefficients $p$, $q$, and $w$ satisfy (4.2) and let the symmetric, coupled, and complex boundary condition be given by, see (4.6),

$$
\mathbf{y}(b)=e^{i \alpha} T \mathbf{y}(a), \quad \text { for some } \alpha \in(-\pi, 0) \cup(0, \pi) ;
$$

let $A$ be the unique selfadjoint, unbounded differential operator in $L^{2}((a, b) ; w)$, specified by (4.1) and (4.6); let the discrete spectrum $\sigma$ of $A$ be represented by $\left\{\lambda_{n}: n \in \mathbb{Z}\right\}$ with $\lim _{n \rightarrow \pm \infty} \lambda_{n}= \pm \infty$, and let $\left\{\psi_{n}: n \in \mathbb{Z}\right\}$ represent the corresponding eigenfunctions. Let the analytic function $D(T, \cdot): \mathbb{C} \rightarrow \mathbb{C}$ be defined by, with solutions $u$, $v$ determined by (4.8),

$$
\begin{aligned}
D(T, \lambda):= & t_{11}[u(\cdot, \lambda), \phi](b)+t_{22}[v(\cdot, \lambda), \theta](b) \\
& -t_{12}[v(\cdot, \lambda), \phi](b)-t_{21}[u(\cdot, \lambda), \theta](b) .
\end{aligned}
$$

Then

(i) $D(T, \cdot) \in \mathbf{H}(\mathbb{C})$;

(ii) $\lambda$ is an eigenvalue of $A$ if and only if $\lambda$ is a zero of $D(T, \lambda)-2 \cos (\alpha)$;

(iii) the zeros of $D(T, \lambda)-2 \cos (\alpha)$ are real and simple;

(iv) the eigenvalues of $A$ are simple.

Let the above-stated definitions and conditions hold; then the boundary value problem (4.1) and (4.6) generate two independent analytic Kramer kernels $K_{1}$ and $K_{2}$ :

$$
\begin{aligned}
& K_{1}(x, \lambda):=\left([u(\cdot, \lambda), \theta](b)-e^{i \alpha} t_{12}\right) v(x, \lambda)-\left([v(\cdot, \lambda), \theta](b)-e^{i \alpha} t_{11}\right) u(x, \lambda), \\
& K_{2}(x, \lambda):=\left([u(\cdot, \lambda), \phi](b)-e^{i \alpha} t_{22}\right) v(x, \lambda)-\left([v(\cdot, \phi), \theta](b)-e^{i \alpha} t_{21}\right) u(x, \lambda) .
\end{aligned}
$$

Proof. See [16].

The real case. The second part of [16] is concerned with real boundary value problems, that is, $\alpha=-\pi, 0, \pi$, for which the following structural condition holds (see Remark 4.5).

Condition 4.7. In the real case $\alpha=-\pi, 0, \pi$, all the eigenvalues are assumed to be simple.

The results in this case are similar to the results stated in Theorem 4.6 except that a phenomenon of degeneracy may occur; see [16, Section 8, Definition 3].

THEOREM 4.8. Let all the conditions of Theorem 4.6 hold with the addition of conditions (4.6); let the kernels $K_{1}$ and $K_{2}$ be given by (4.11) and the phenomenon of degeneracy be defined as in [16]. For $r=1,2$, let the subspaces $L_{r}^{2}((a, b) ; w)$ of $L^{2}((a, b) ; w)$ be defined by

$$
L_{r}^{2}((a, b) ; w):=\operatorname{span}\left\{\psi_{n} ; n \in \mathbb{Z}_{r}\right\} \quad(r=1,2)
$$

Then

(i) every eigenvalue in $\left\{\lambda_{n}: n \in \mathbb{Z}\right\}$ is nondegenerate for at least one $K_{r}$;

(ii) for $r=1,2$, the kernel $K_{r}$ is an analytic Kramer kernel for the subspace $L_{r}^{2}((a, b) ; w)$;

(iii) $K(x, \lambda)=\alpha_{1} K_{1}(x, \lambda)+\alpha_{2} K_{2}(x, \lambda)(x \in(a, b) ; \lambda \in \mathbb{C})$ is an analytic Kramer kernel for the whole space $L^{2}((a, b) ; w)$, for $\alpha_{1}, \alpha_{2} \in \mathbb{R}$. 
Proof. See [16].

Remark 4.9. The case when the multiplicity of the spectrum $\sigma(A)$ is 2 is fully examined in [17].

Examples for both the above complex and real cases can be found in [16] and also in $[15$, Section 5.2]. In all the examples, the regular differential equation

$$
-y^{\prime \prime}(x)=\lambda y(x) \quad(x \in[-\pi, \pi])
$$

is considered and $\theta(x)=\cos x$ and $\phi(x)=\sin x$ are chosen so as to give the boundary conditions (see (4.6))

$$
\mathbf{y}(\pi) \equiv\left(\begin{array}{c}
y^{\prime}(\pi) \\
-y(\pi)
\end{array}\right)=e^{i \alpha} T\left(\begin{array}{c}
y^{\prime}(-\pi) \\
-y(-\pi)
\end{array}\right) \equiv e^{i \alpha} T \mathbf{y}(-\pi)
$$

The functions $u$ and $v$ that satisfy the initial conditions are

$$
\begin{aligned}
& u(x, \lambda)=-\cos (\sqrt{\lambda}(x+\pi)), \\
& v(x, \lambda)=\frac{1}{\sqrt{\lambda}} \sin (\sqrt{\lambda}(x+\pi)) .
\end{aligned}
$$

\section{Acknowledgment}

The author wishes to express her gratitude to Professor W. N. Everitt for his help, support, advice, and for his guidance in the area of research that concerns this paper.

\section{References}

[1] N. I. Akhiezer and I. M. Glazman, Theory of Linear Operators in Hilbert Space. Vol. I, Monographs and Studies in Mathematics, vol. 9, Pitman, Massachusetts, 1981.

[2] _ Theory of Linear Operators in Hilbert Space. Vol. II, Monographs and Studies in Mathematics, vol. 10, Pitman, Massachusetts, 1981.

[3] P. B. Bailey, W. N. Everitt, and A. Zettl, Regular and singular Sturm-Liouville problems with coupled boundary conditions, Proc. Roy. Soc. Edinburgh Sect. A 126 (1996), no. 3, 505-514.

[4] P. L. Butzer, A survey of the Whittaker-Shannon sampling theorem and some of its extensions, J. Math. Res. Exposition 3 (1983), no. 1, 185-212.

[5] P. L. Butzer, W. Splettstösser, and R. L. Stens, The sampling theorem and linear prediction in signal analysis, Jahresber. Deutsch. Math.-Verein. 90 (1988), no. 1, 1-70.

[6] P. L. Butzer and R. L. Stens, Sampling theory for not necessarily band-limited functions: a historical overview, SIAM Rev. 34 (1992), no. 1, 40-53.

[7] L. L. Campbell, A comparison of the sampling theorems of Kramer and Whittaker, J. Soc. Indust. Appl. Math. 12 (1964), 117-130.

[8] N. Dunford and J. T. Schwartz, Linear Operators. Part II: Spectral Theory. Self Adjoint Operators in Hilbert Space, John Wiley \& Sons, New York, 1963.

[9] W. N. Everitt, Linear ordinary quasidifferential expressions, Proceedings of the 1983 Beijing Symposium on Differential Geometry and Differential Equations, Science Press, Beijing, 1986, pp. 1-28. 
[10] W. N. Everitt, W. K. Hayman, and G. Nasri-Roudsari, On the representation of holomorphic functions by integrals, Appl. Anal. 65 (1997), no. 1-2, 95-102.

[11] W. N. Everitt and L. Markus, Controllability of [r]-matrix quasi-differential equations, J. Differential Equations 89 (1991), no. 1, 95-109.

[12] , The Glazman-Krein-Naimark theorem for ordinary differential operators, New Results in Operator Theory and Its Applications, Oper. Theory Adv. Appl., vol. 98, Birkhäuser, Basel, 1997, pp. 118-130.

[13]__ Boundary Value Problems and Symplectic Algebra for Ordinary Differential and QuasiDifferential Operators, Mathematical Surveys and Monographs, vol. 61, American Mathematical Society, Rhode Island, 1999.

[14] Complex symplectic geometry with applications to ordinary differential operators, Trans. Amer. Math. Soc. 351 (1999), no. 12, 4905-4945.

[15] W. N. Everitt and G. Nasri-Roudsari, Interpolation and sampling theories, and linear ordinary boundary value problems, Sampling Theory in Fourier and Signal Analysis, Advanced Topics, vol. 5, Oxford University Press, Oxford, 1999, pp. 96-129.

[16] Sturm-Liouville problems with coupled boundary conditions and Lagrange interpolation series, J. Comput. Anal. Appl. 1 (1999), no. 4, 319-347.

[17] Sturm-Liouville problems with coupled boundary conditions and Lagrange interpolation series. II, Rend. Mat. Appl. (7) 20 (2000), 199-238.

[18] W. N. Everitt, G. Nasri-Roudsari, and J. Rehberg, A note on the analytic form of the Kramer sampling theorem, Results Math. 34 (1998), no. 3-4, 310-319.

[19] W. N. Everitt and A. Poulkou, Kramer analytic kernels and first-order boundary value problems, J. Comput. Appl. Math. 148 (2002), no. 1, 29-47.

[20] W. N. Everitt and D. Race, Some remarks on linear ordinary quasidifferential expressions, Proc. London Math. Soc. (3) 54 (1987), no. 2, 300-320.

[21] W. N. Everitt, G. Schöttler, and P. L. Butzer, Sturm-Liouville boundary value problems and Lagrange interpolation series, Rend. Mat. Appl. (7) 14 (1994), no. 1, 87-126.

[22] W. N. Everitt and A. Zettl, Generalized symmetric ordinary differential expressions. I. The general theory, Nieuw Arch. Wisk. (3) 27 (1979), no. 3, 363-397.

[23] - Differential operators generated by a countable number of quasi-differential expressions on the real line, Proc. London Math. Soc. (3) 64 (1992), no. 3, 524-544.

[24] M. Genuit and G. Schöttler, A problem of L. L. Campbell on the equivalence of the Kramer and Shannon sampling theorems, Comput. Math. Appl. 30 (1995), no. 3-6, 433-443.

[25] I. Halperin, Closures and adjoints of linear differential operators, Ann. of Math. (2) 38 (1937), no. 4, 880-919.

[26] J. R. Higgins, Five short stories about the cardinal series, Bull. Amer. Math. Soc. (N.S.) 12 (1985), no. $1,45-89$.

[27] Sampling Theory in Fourier and Signal Analysis, Foundations, Oxford University Press, Oxford, 1996.

[28] J. R. Higgins and R. Stens, Sampling Theory in Fourier and Signal Analysis, Advanced Topics, vol. 2, Oxford University Press, Oxford, 1999.

[29] A. J. Jerri, On the equivalence of Kramer's and Shannon's sampling theorems, IEEE Trans. Information Theory IT-15 (1969), 497-499.

[30] _ The Shannon sampling theorem-its various extensions and applications: A tutorial review, Proc. IEEE (1977), no. 11, 1565-1596.

[31] V. A. Kotel'nikov, On the carrying capacity of the ether and wire in telecommunications, Material for the First All-Union Conference on Questions of Communication, Izd. Red. Upr. Svyazi RKKA, Moskow, 1933 (Russian).

[32] H. P. Kramer, A generalized sampling theorem, J. Math. Phys. 38 (1959), 68-72. 
[33] R. J. Marks II, Introduction to Shannon Sampling and Interpolation Theory, Springer Texts in Electrical Engineering, Springer-Verlag, New York, 1991.

[34] M. A. Naŭmark, Linear Differential Operators. Part II: Linear Differential Operators in Hilbert Space, Frederick Ungar, New York, 1968, with additional material by the author, and a supplement by V. È. Ljance. Translated from the Russian by E. R. Dawson. English translation edited by W. N. Everitt.

[35] C. E. Shannon, Communication in the presence of noise, Proc. IRE 37 (1949), 10-21.

[36] D. Shin, Existence theorems for the quasi-differential equation of the nth order, Dokl. Akad. Nauk SSSR 18 (1938), 515-518.

[37] On the quasi-differential transformations in Hilbert space, Dokl. Akad. Nauk SSSR 18 (1938), 523-526.

[38] On the solutions of a linear quasi-differential equation of order n, Mat. Sb. 7 (1940), 479-532.

[39] E. C. Titchmarsh, Eigenfunction Expansions Associated with Second-Order Differential Equations. Part I, 2nd ed., Clarendon Press, Oxford University Press, 1962.

[40] E. T. Whittaker, On the functions which are represented by the expansions of the interpolation theory, Proc. Roy. Soc. Edinburgh 35 (1915), 181-194.

[41] A. I. Zayed, On Kramer's sampling theorem associated with general Sturm-Liouville problems and Lagrange interpolation, SIAM J. Appl. Math. 51 (1991), no. 2, 575-604.

[42] A. I. Zayed, G. Hinsen, and P. L. Butzer, On Lagrange interpolation and Kramer-type sampling theorems associated with Sturm-Liouville problems, SIAM J. Appl. Math. 50 (1990), no. 3, 893-909.

[43] A. Zettl, Formally self-adjoint quasi-differential operators, Rocky Mountain J. Math. 5 (1975), 453-474.

Anthippi Poulkou: Department of Mathematics, National and Capodistrian University of Athens, Panepistimioupolis, 15784 Athens, Greece

E-mail address: apoulkou@cc.uoa.gr 


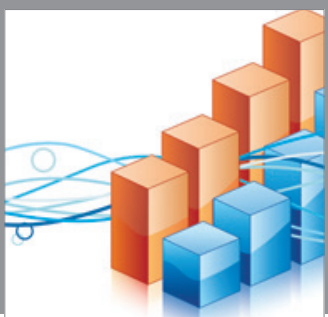

Advances in

Operations Research



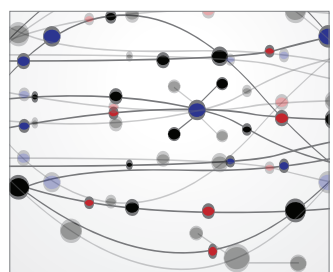

\section{The Scientific} World Journal
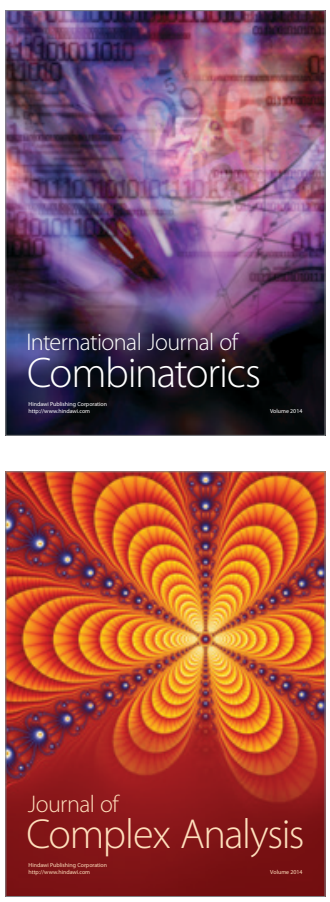

International Journal of

Mathematics and

Mathematical

Sciences
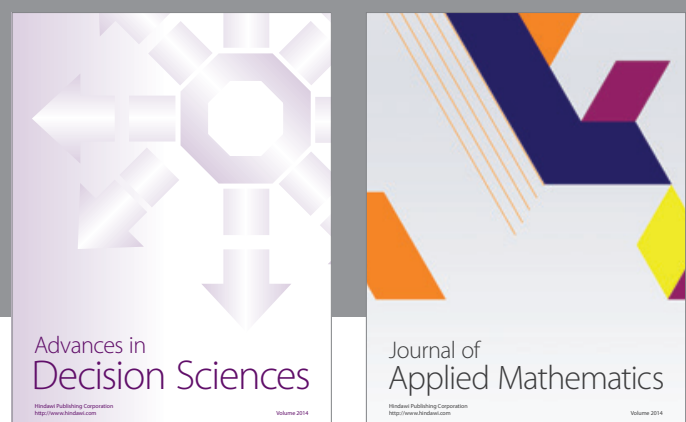

Journal of

Applied Mathematics
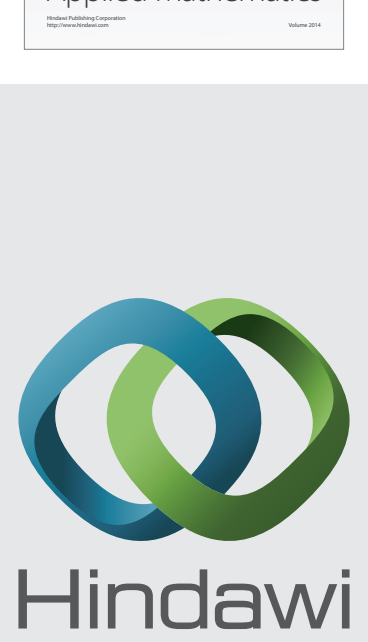

Submit your manuscripts at http://www.hindawi.com
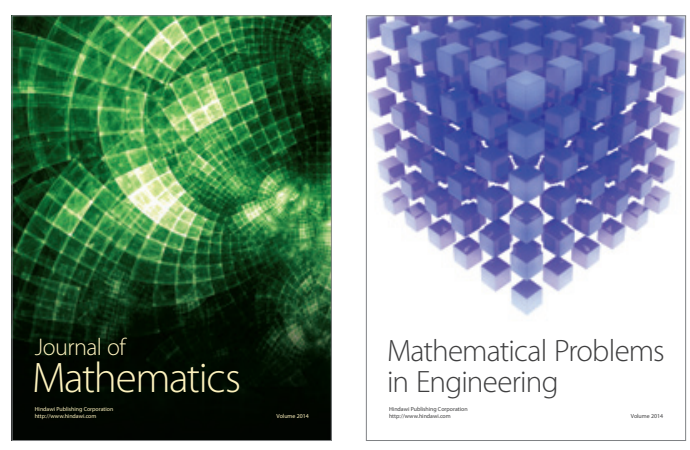

Mathematical Problems in Engineering
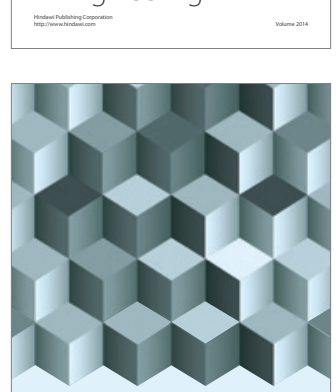

Journal of

Function Spaces
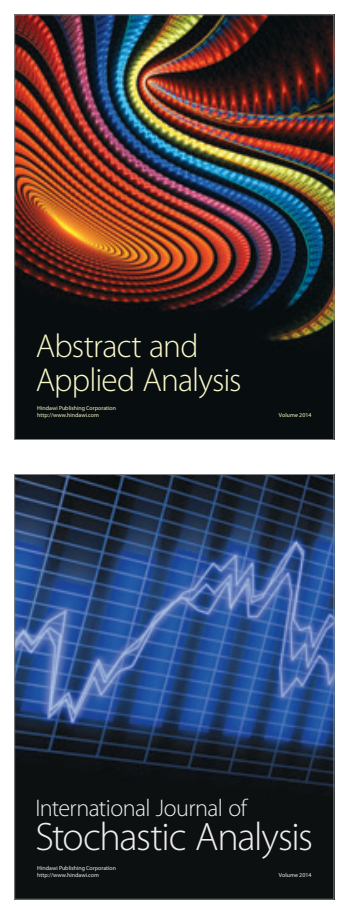

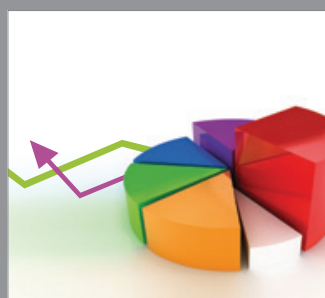

ournal of

Probability and Statistics

Promensencen
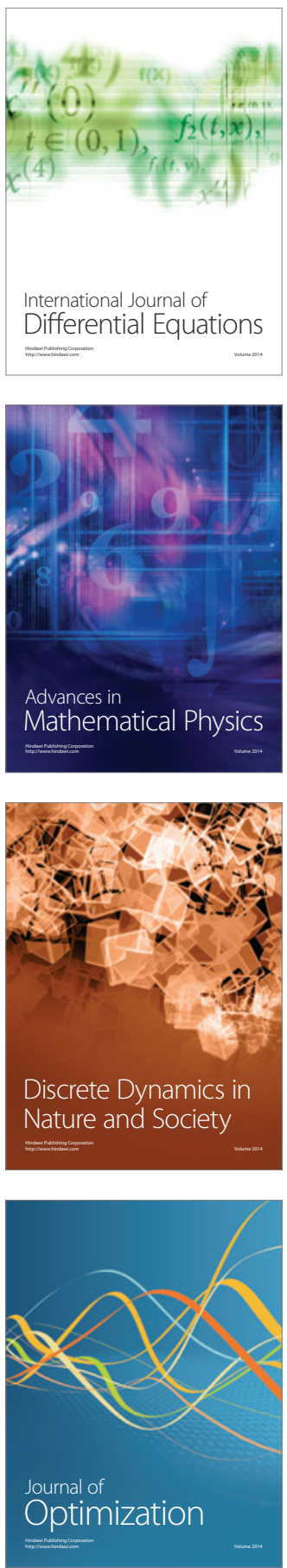